\title{
Le Paris musical comme expérience de savoir : ma période d'études et de doctorat à l'EHESS (2002-2008)
}

\author{
Gesa zur Nieden \\ Traducteur : Michelle Biget-Mainfroy
}

\section{(2) OpenEdition}

\section{Journals}

Édition électronique

URL : http://journals.openedition.org/transposition/1804

DOI : 10.4000/transposition. 1804

ISSN : 2110-6134

\section{Éditeur}

CRAL - Centre de recherche sur les arts et le langage

\section{Référence électronique}

Gesa zur Nieden, «Le Paris musical comme expérience de savoir : ma période d'études et de doctorat à l'EHESS (2002-2008) », Transposition [En ligne], Hors-série 1 | 2018, mis en ligne le 30 janvier 2018, consulté le 11 février 2020. URL : http://journals.openedition.org/transposition/1804 ; DOI : 10.4000/ transposition. 1804

Ce document a été généré automatiquement le 11 février 2020.

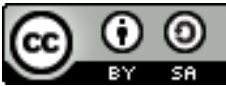

La revue Transposition est mise à disposition selon les termes de la Licence Creative Commons Attribution - Partage dans les Mêmes Conditions 4.0 International. 


\title{
Le Paris musical comme expérience de savoir : ma période d'études et de doctorat à l'EHESS (2002-2008)
}

\author{
Gesa zur Nieden \\ Traduction : Michelle Biget-Mainfroy
}

1 Lorsque je pénétrai pour la première fois dans la Maison des sciences de l'homme, je fus frappée par l'architecture aujourd'hui familière de la cage d'escalier, encadrée par des ascenseurs. En dépit de la situation prestigieuse de l'École rive gauche et au cœur du $6^{\mathrm{e}}$ arrondissement, elle me rappelait un peu mon université d'origine de Bochum. Après deux années d'études à Venise et à l'université de Paris IV-Sorbonne, je me rendais ce jour-là à un entretien pour m'inscrire dans le nouveau cursus «Musique, histoire, société ", accompagnée par une amie mexicaine qui préparait déjà son doctorat à l'EHESS et m'avait, comme beaucoup de résidents côtoyés à la Cité internationale universitaire de Paris, recommandé l'École. Lorsque je ressortis de l'entretien, je tenais en mains un bout de papier sur lequel mon futur directeur de thèse, Michael Werner, avait noté le sujet de mon travail - et ce titre devait rester à peu près le même durant toute la durée de mon DEA puis de mon doctorat ${ }^{1}$.

2 Je me souviens des cours et des opportunités d'échange à l'EHESS, dès le début, comme de sites et d'espaces motivant l'intérêt de personnes venues de différents pays et propices au débat international. Il en était ainsi pour les étudiants mais aussi pour les enseignants et enseignantes qui avaient entrepris ce projet de présenter à de nouvelles générations de chercheurs Paris comme une ville musicale et comme un lieu institutionnalisé de savoir ouvert sur l'extérieur. Nous pouvions ici avoir accès à de multiples démarches : un cours d'introduction à la musicologie dans la tradition de Paris IV, un cycle de cours magistraux sur la musique électro-acoustique à l'IRCAM, des séminaires autour de controverses internationales actuelles commeles capacités narratives de la musique, un cours sur La Nuit transfigurée de Schönberg croisant l'histoire du droit et celle des représentations esthétiques ${ }^{2}$, une conférence sur l'intégration mutuelle des infrastructures musicales de Berlin-Est et Berlin-Ouest après 
1989, des séances au Conservatoire Supérieur sur l'historiographie de l'écriture scientifique chez Fétis ${ }^{3}$, une conférence sur " la musique de la conception du monde » prononcée par un professeur invité de Berlin ${ }^{4}$ dans le cadre d'un séminaire de l'ENS... $\mathrm{Au}$ premier séminaire auquel j'assistai, je me trouvais assise à côté d'un musicologue qui venait de commencer sa thèse sur le genre du Noël dans le Sud de la France au XVIII siècle et m'interrogea aussitôt sur toutes les définitions possibles de concepts, proposant ainsi de mettre les siennes en discussion. C'était comme si la ville de Paris et son offre de musiques les plus diverses trouvait là valeur de pratique de connaissance. Parallèlement, nous avions un accès direct à des activités internationales de premier plan coordonnées par l'EHESS ou par le Centre interdisciplinaire d'études et de recherches sur l'Allemagne (CIERA). Globalement, beaucoup de choses étaient en jeu dans cette conception du cursus croisant les institutions et mettant l'accent sur une dimension internationale, dont témoignaient les premiers travaux de fin d'études et les thèses - et peut-être même que nous, étudiants et doctorants, participions aussi d'un nouveau type de travail interdisciplinaire sur la musique en France. Nous écoutions beaucoup - et étions nous-mêmes écoutés. Il s'agissait de questionner sur un mode pluridisciplinaire les conventions en vigueur en matière d'écriture de l'histoire de la musique et de découvrir de nouvelles perspectives en intégrant la sociologie, l'anthropologie et l'ethnologie. Ce qui fut et demeure pour moi le plus important des acquis durant ce temps d'études et de préparation de mon doctorat, c'est la réflexion du chercheur sur son positionnement et l'élargissement constant à différents regards sur les contextes, pratiques et événements musicaux.

De cette expérience résultèrent des contacts échelonnés sur des années, qui rendirent possibles et même quotidiens des projets binationaux, franco-allemands et également internationaux. Un colloque organisé à Berlin avec Talia Bachir-Loopuyt, Sara Iglesias et Anna Langenbruch sur le thème " Musique - contextes " permit d'élargir le cercle franco-allemand des jeunes chercheurs travaillant sur la musique, dans une perspective à la fois internationale et interdisciplinaire ${ }^{5}$. Alors que j'étais chargée de recherche à l'Institut Historique Allemand de Rome, une autre collaboration se tissa avec l'École française de Rome, débouchant sur un projet ANR-DFG sur les musiciens européens à Venise, Rome et Naples entre 1650 et 1750 : la première coopération entre historiens de la musique de ces deux institutions depuis plusieurs décennies ${ }^{6}$. Parmi les jeunes musicologues avec lesquels je préparais mon doctorat, nombreux sont ceux qui participent aujourd'hui à un manuel en allemand intitulé Lieux et espaces de la musique, 1740-19147. Les préparatifs de ce volume mettent en lumière ce qui a nourri et caractérisé notre génération : l'intérêt pour la transversalité de méthodes, de concepts et de catégories, avec un accent sur la systématisation mais aussi le traitement méticuleux des sources. Ces trois composantes, nous les avons rencontrées durant notre cursus scientifique sous différentes formes, tant auprès des autres collègues que des institutions fréquentées, et nous nous efforçons de les remettre en permanence en discussion afin de pouvoir les traiter de manière équitable. Dans les mêmes temps, l'ouverture de l'histoire de la musique sur les récentes orientations sociologiques, philosophiques ou anthropologiques, ainsi qu'elle nous a été enseignée par le biais des approches en termes de transferts culturels et d'histoire croisée, se confirme aussi lentement mais sûrement. En 2016, j'ai présenté avec deux collègues allemandes une communication au congrès annuel de la Gesellschaft für Musikforschung (Société allemande de musicologie), dans laquelle nous sommes revenues sur nos recherches doctorales pour mettre en évidence des chevauchements théoriques et 
méthodologiques entre histoire et sociologie de la musique ${ }^{8}$. Actuellement, je travaille à une ethnographie de la réception de Richard Wagner depuis 1945. Grâce au dialogue que je poursuis avec des anciens de l'EHESS, qui a par exemple donné lieu à un panel franco-allemand sur les liens entre ethnographie et histoire de la musique lors du congrès de la Gesellschaft für Musikforschung en 2017 à Kassel, cette recherche s'intègre en même temps dans une discussion plus large sur les études musicologiques. La quête permanente et quasi naturelle d'échanges avec des collègues d'autres disciplines, et la relativisation du point de vue du chercheur qui en découle peuvent sembler déconcertantes pour qui tente de se faire une place dans la musicologie allemande. Mais cela garantit précisément le dynamisme de la recherche et permet que surgissent de nouvelles voies et passerelles'. C'est ainsi que ce temps passé à l'EHESS, qui m'a beaucoup enrichie et marquée, demeure pour moi jusqu'à aujourd'hui une sorte de Heimat, un foyer scientifique. Et ce ressenti perdure lorsque je gravis les escaliers du bâtiment construit dans le style des années 70 de ma présente université, lorsque je voyage en Europe de l'Est pour des congrès ou lorsque je tente de partager avec mes étudiants une réflexion intégrant des perspectives multiples et de les faire accéder à d'autres cultures de recherche.

\section{BIBLIOGRAPHIE}

BACHIR-LOOPUYT, Talia, LANGENBRUCH, Anna, IGLESIAS, Sara et NIEDEN, Gesa zur (eds), Musik Kontext - Wissenschaft: Interdisziplinäre Forschung zu Musik/Musiques - contextes - savoirs :

Perspectives interdisciplinaires sur la musique, Francfort, Peter Lang, 2012.

BUCH, Esteban, Le cas Schönberg. Naissance de l'avant-garde musicale, Paris, Gallimard 2006.

CAMPOS Rémy, François-Joseph Fétis : Musicographe, Genève, Droz 2013.

DANUSER, Hermann, Weltanschauungsmusik, Schliengen, Argus, 2009.

GOULET, Anne-Madeleine et NIEDEN, Gesa zur (eds), Europäische Musiker in Venedig, Rom und Neapel / Les musiciens européens à Venise, Rome et Naples / Musicisti europei a Venezia, Roma e Napoli 1650-1750, Kassel, Bärenreiter, 2015.

LANGENBRUCH, Anna et NIEDEN, Gesa zur (eds), Handbuch der Musik der Klassik und der Romantik, Laaber-Verlag, à paraître en 2018.

NIEDEN, Gesa zur, Vom Grand Spectacle zur Great Season. Das Pariser Théâtre du Châtelet als Raum musikalischer Produktion und Rezeption (1862-1914), Vienne/Cologne/Weimar, Böhlau/oldenbourg, 2010.

NIEDEN, Gesa zur et OVER, Berthold (eds), Musicians' Mobilities and Music Migrations in Early Modern Europe. Biographical Patterns and Cultural Exchanges, Bielefeld, transcript, 2016. 


\section{NOTES}

1. NIEDEN, Gesa zur, Du grand spectacle à la great season. Le Théâtre du Châtelet comme espace de production musicale (1862-1909), Diplôme d'Études Approfondies à l'École des hautes études en sciences sociales de Paris (Michael Werner, Patrice Veit), soutenu en 2003 ; id., Vom grand spectacle zur great season. Das Pariser Théâtre du Châtelet als Raum musikalischer Produktion und Rezeption (1862-1914), Thèse de doctorat réalisée en co-tutelle à l'École des hautes études en sciences sociales de Paris (Michael Werner) et à l'Université de la Ruhr à Bochum (Monika Woitas), soutenue en juillet 2008. Publiée : NIEDEN, Gesa zur, Vom Grand Spectacle zur Great Season. Das Pariser Théâtre du Châtelet als Raum musikalischer Produktion und Rezeption (1862-1914), Vienne/Cologne/Weimar, Böhlau/Oldenbourg, 2010.

2. BUCH, Esteban, Le cas Schönberg. Naissance de l'avant-garde musicale, Paris, Gallimard 2006.

3. CAMPOS, Rémy, François-Joseph Fétis : Musicographe, Genève, Droz 2013.

4. DANUSER, Hermann, Weltanschauungsmusik, Schliengen, Argus, 2009.

5. Voir le compte-rendu de Katja Bethe sur http://hsozkult.geschichte.hu-berlin.de/ index.asp?id=3415\&view=pdf\&pn=tagungsberichte\&type=tagungsberichte (consulté le 27/2/2018) ainsi que les actes du colloque : BACHIR-LOOPUYT Talia, LANGENBRUCH, Anna, IGLESIAS, Sara et NIEDEN, Gesa zur (eds), Musik - Kontext - Wissenschaft: Interdisziplinäre Forschung zu Musik / Musiques - contextes - savoirs: Perspectives interdisciplinaires sur la musique, Francfort, Peter Lang, 2012.

6. Projet ANR-DFG «Musici »(2010-2013), en coopération avec Anne-Madeleine Goulet. Cf. www.musici.eu De ce projet ont émergé deux autres coopérations sur la mobilité de musiciens à l'époque moderne: un projet européen financé par le fonds HERA (Humanities in the European Research Area) en coopération avec des collègues de Berlin, Ljubljana, Varsovie et Zagreb (2013-2016, www.musmig.eu); et un projet DFG-NCN en coopération avec Aneta Markuszewska de l'Université de Varsovie (« Pasticcio », 2018-2020).

7. LANGENBRUCH, Anna et NIEDEN, Gesa zur (eds), Handbuch der Musik der Klassik und der Romantik, Laaber-Verlag, à paraître en 2018.

8. LANGENBRUCH, Anna, STAHRENBERG, Carolin et NIEDEN, Gesa zur, communication «Aufführungsorte, Handlungsräume, Musik-Topographien: Raumsoziologische Perspektiven auf Musikgeschichte » dans la section «Zuständigkeiten der Musiksoziologie », Jahrestagung der Gesellschaft für Musikforschung, Mainz, 14 septembre 2016.

9. Cf. par exemple mes deux projets de recherche Musici et MusMig: GOULET, AnneMadeleine et NIEDEN, Gesa zur (eds), Europäische Musiker in Venedig, Rom und Neapel / Les musiciens européens à Venise, Rome et Naples / Musicisti europei a Venezia, Roma e Napoli 1650-1750, Kassel, Bärenreiter, 2015; NIEDEN, Gesa zur et OVER, Berthold (eds), Musicians' Mobilities and Music Migrations in Early Modern Europe. Biographical Patterns and Cultural Exchanges, Bielefeld, transcript, 2016. 


\section{AUTEURS}

\section{GESA ZUR NIEDEN}

Gesa zur Nieden est depuis 2011 Professeur Junior de musicologie à l'Université Johannes Gutenberg à Mayence. Après des études en études théâtrales, littérature comparée et musicologie à Bochum, Venise et Paris, elle a réalisé une thèse en cotutelle à l'EHESS de Paris et l'Université de Bochum sur le Théâtre du Châtelet comme lieu de production et réception musicale. De 2008 à 2011, elle a été chargée de recherche à l'Institut Historique Allemand de Rome où elle a dirigé un projet ANR-DFG en collaboration avec Anne Madeleine Goulet et l'École française de Rome. Entre 2013 et 2016, elle s'est associée à un projet européen HERA sur les migrations de musiciens à l'époque moderne avec des collègues de Berlin, Ljubljana, Varsovie et Zagreb. Professeur invitée à l'Université de Cologne en 2016-2017, elle dirige aujourd'hui avec Aneta Markuszewska (Université de Varsovie) un projet de recherche germano-polonais sur le pastiche d'opéra au $\mathrm{XVIII}^{\mathrm{e}}$ siècle. Parmi ses publications récentes figure un livre édité avec Berthold Over, Musicians' Mobilities and Music Migrations in Early Modern Europe. Biographical Patterns and Cultural Exchanges (transcript, 2016). 